Article

\title{
Synthesis and Pharmacological Activities of Some New Triazolo- and Tetrazolopyrimidine Derivatives
}

\author{
Saleh A. Bahashwan ${ }^{1}$, Ahmed A. Fayed ${ }^{2,3, *}$, Abd El-Galil E. Amr ${ }^{3,4}$, Eman M. Flefel ${ }^{3}$ and \\ Atef Kalmouch $^{3,5}$
}

1 Pharmacology and Toxicology Department, College of Pharmacy, Taibah University, Al-Madinah Al-Munawara 3893, Saudi Arabia

2 Respiratory Department (Applied Organic \& Biochemistry Division), College of Medical Rehabilitation Sciences, Taibah University, Al-Madinah Al-Munawara 3893, Saudi Arabia

3 National Research Center, Dokki, Cairo, Dokki 12622, Egypt

4 Pharmaceutical Chemistry Department, Drug Exploration \& Development Chair (DEDC), College of Pharmacy, King Saud University, Riyadh 11451, Saudi Arabia

5 Deanship of Academic Services Preparatory Years, Health Science Track, Taibah University, Al Madinah Al-Monawarah 344, Saudi Arabia

* Author to whom correspondence should be addressed; E-Mail: dr_ahmedfayed14@yahoo.com; Fax: +966-11-4673-768.

Received: 27 September 2013; in revised form: 21 November 2013 / Accepted: 29 November 2013 / Published: 6 December 2013

\begin{abstract}
A new series of fused triazolo- and tetrazolopyrimidine derivatives 2-14 were synthesized and their anti-inflammatory and ulcerogenic activities were evaluated. The pharmacological screening showed that many of these obtained compounds have good anti-inflammatory activities, comparable to the reference drug. The toxicity of the compounds was also assayed via the determination of their $\mathrm{LD}_{50}$ values. The structures of newly synthesized compounds were confirmed by IR, ${ }^{1} \mathrm{H}-\mathrm{NMR}$, MS spectral data and elemental analysis.
\end{abstract}

Keywords: pyrazolopyrimidines; thienopyrimidines; triazolo- and tetrazolopyrimidines; anti-inflammatory and ulcerogenic activities 


\section{Introduction}

In our previous work, some polycyclic heterocyclic derivatives were studied as $5 \alpha$-reductase inhibitors, antiviral and anti-tumor agents [1]. Some of these compounds also showed aromatase and quinone reductase-2 inhibitors [2], anti-inflammatory, analgesic and antipyretic [3,4], and anti-arthritic and immunosuppressive activities [5]. Pyrimidines and fused pyrimidines, being integral parts of DNA and RNA, play an essential role in several biological processes and also have considerable chemical and pharmacological importance as antibiotics, antibacterials, cardiovascular as well as agrochemical and veterinary products [6-12]. Heterocyclic compounds play an important role in designing new classes of structural entities of medicinal importance with potentially new mechanisms of action. These heterocyclic compounds are well known to possess diverse pharmacological properties, viz. antimicrobial, analgesic, anti-inflammatory, anticancer, anticonvulsant and antimalarial activities [13]. On the other hand, we have reported that some of our new substituted heterocyclic compounds exhibited antiparkinsonian [14], antitumor [15,16], and anti-inflammatory [17] activities. In addition, during the last few years, condensed thienopyrimidine derivatives have received considerable attention. Many of these derivatives were found to possess a variety of pronounced activities such as anti-inflammatory and analgesic [18-21], antimicrobial [22-25], anti-avian influenza virus (H5N1) [26], anti-herpes simplex virus type-1 (HSV-1) and hepatitis-A virus (HAV), serotonin 5-HT 6 receptor antagonist [27], antiarrhythmic [28] agent properties. Pyrimidine derivatives have been previously reported as platelet aggregation inhibitors, antagonists, anti-conceptive and anti-parkinsonism [29-32] agents. Heterocyclic compounds have also exhibited anthelmintic, anti HIV activity and hypoglycemic activities [33]. In view of these observations and as continuation of our previous works on heterocyclic chemistry, we report herein the synthesis of some new heterocyclic containing pyrazolothienopyrimidine moieties and the study of their anti-inflammatory activities in comparison to indomethacin used as positive control.

\section{Results and Discussion}

\subsection{Chemistry}

2-(4-Methoxypheyl)-4-chlorocycloocteno[4,5]thieno[2,3-e]pyrimidine (1) [34] was treated with hydrazine hydrate to afford the corresponding hydrazinopyrimidine $\mathbf{2}$. Reaction of compound $\mathbf{1}$ with cyanoacetylhydrazine in boiling butanol afforded the corresponding 3-(cyanomethyl)triazolopyrimidine 3. Compound $\mathbf{1}$ was also reacted with amino acids to give imidazo derivatives $\mathbf{4}$ and $\mathbf{5}$, respectively. Treatment of $\mathbf{1}$ with sodium azide in boiling acetic acid gave the corresponding tetrazolopyrimidine 6 (Scheme 1). On the other hand, when compound 2 was refluxed with diethyl oxalate or chloroacetylchloride it afforded triazino derivatives $\mathbf{7}$ and $\mathbf{8}$, respectively. The IR and ${ }^{1} \mathrm{H}-\mathrm{NMR}$ spectra of $\mathbf{7}$ and $\mathbf{8}$ revealed the absence of the $\mathrm{NH}_{2}$ group. Also, reaction of compound $\mathbf{2}$ with ethyl chloroformate or acetic anhydride gave triazolopyrimidine derivatives 9 and 10, respectively. The IR spectrum of compound 9 showed absorption bands due to $\mathrm{C}=\mathrm{O}$ and $\mathrm{NH}$; its ${ }^{1} \mathrm{H}-\mathrm{NMR}$ showed a singlet at $6.24 \mathrm{ppm}$ due to the $\mathrm{NH}$ (amide) group, while the ${ }^{1} \mathrm{H}-\mathrm{NMR}$ of compound $\mathbf{1 0}$ showed a singlet at $2.16 \mathrm{ppm}$ due to the $\mathrm{CH}_{3}$ group (Scheme 2). 
Scheme 1. Synthetic route for compounds 2-6.
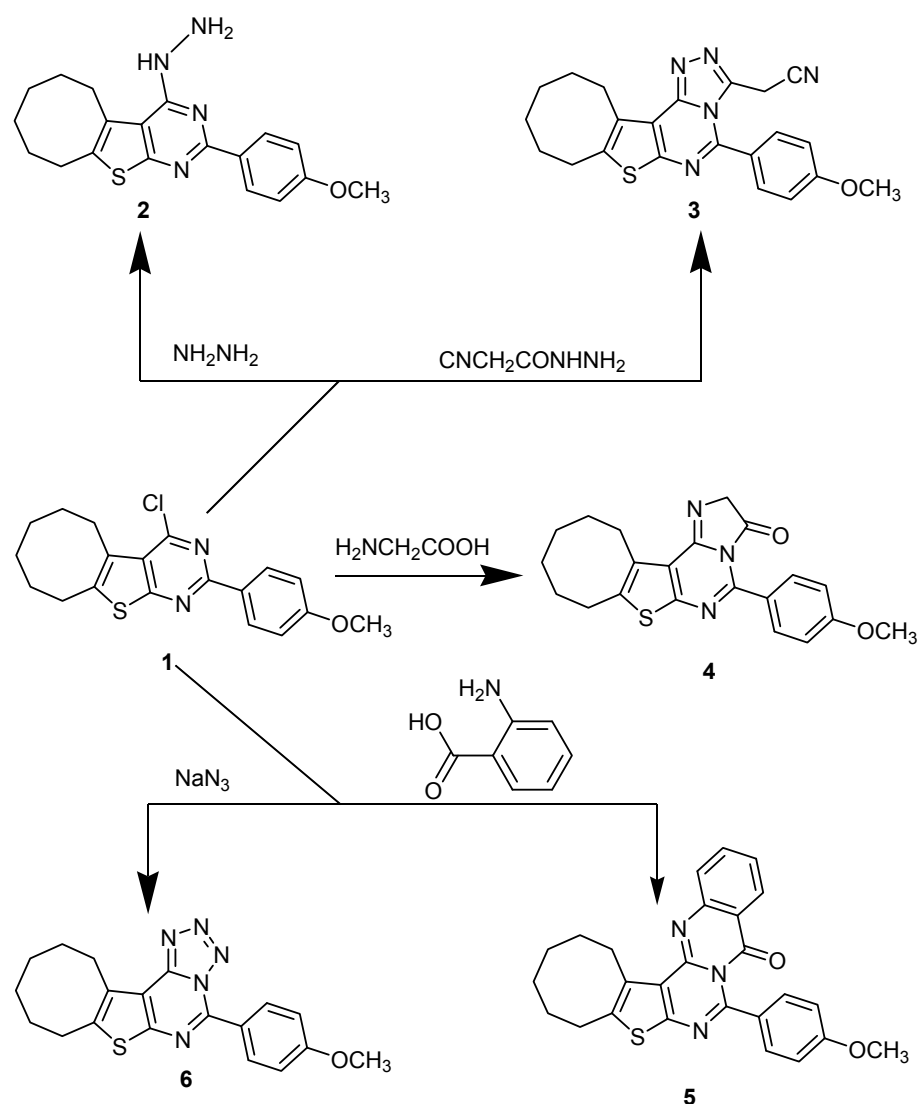

Scheme 2. Synthetic route for compounds 7-10.

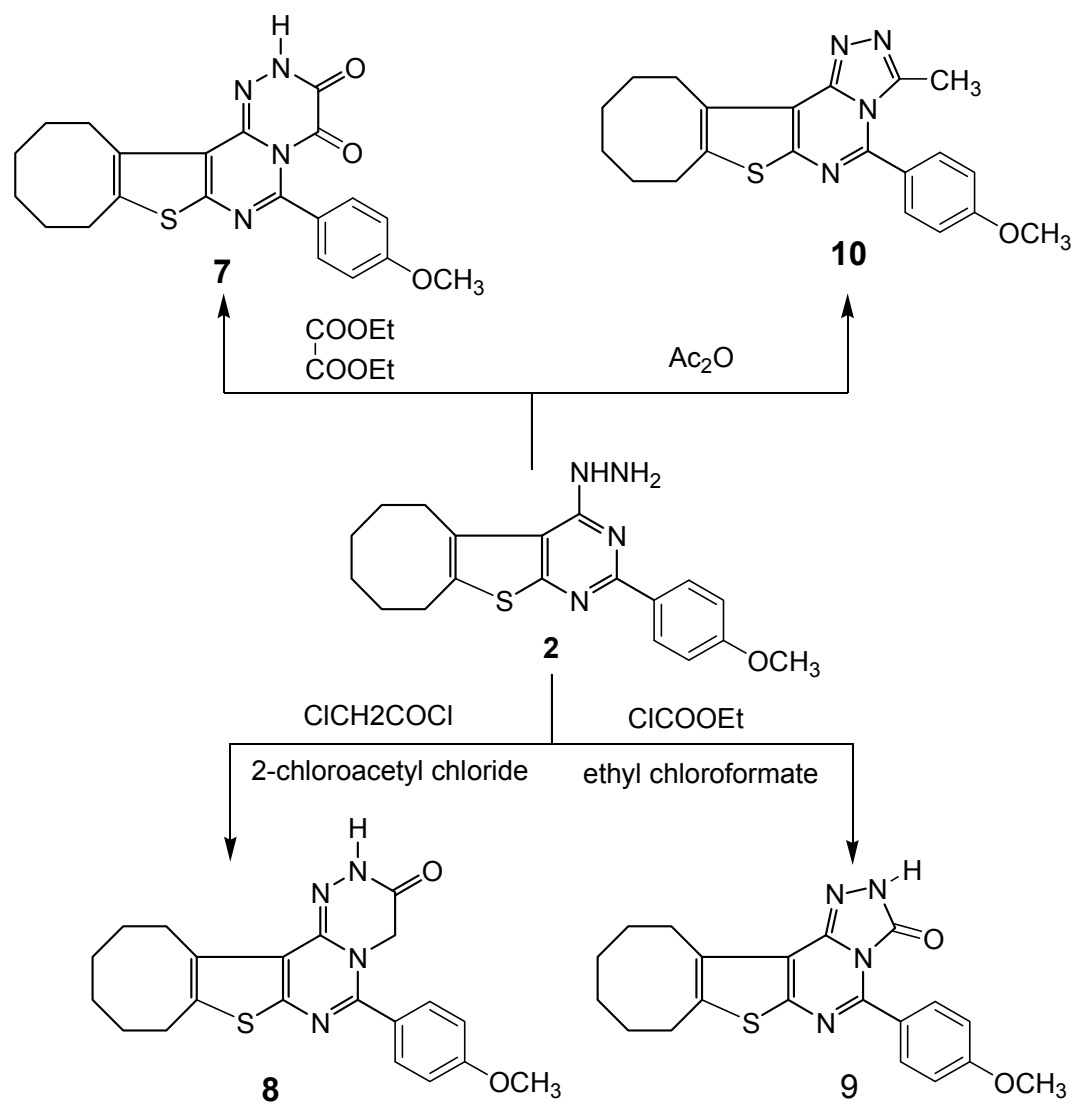


Reaction of compound $\mathbf{2}$ with phenyl isothiocyanate in boiling ethanol yielded compound 11, which was treated with methanolic sodium hydroxide to give the cyclized compound $\mathbf{1 2}$. The ${ }^{1} \mathrm{H}-\mathrm{NMR}$ spectrum of compound $\mathbf{1 2}$ showed a singlet at $\delta=10.23 \mathrm{ppm}$ due to the NH group. Finally, compound 2 was reacted with benzaldehyde to give arylidenehydrazine derivative 13, which was cyclized with bromine and sodium carbonate to give triazolopyrimldine 14 (Scheme 3 ).

Scheme 3. Synthetic route for compounds 11-14.

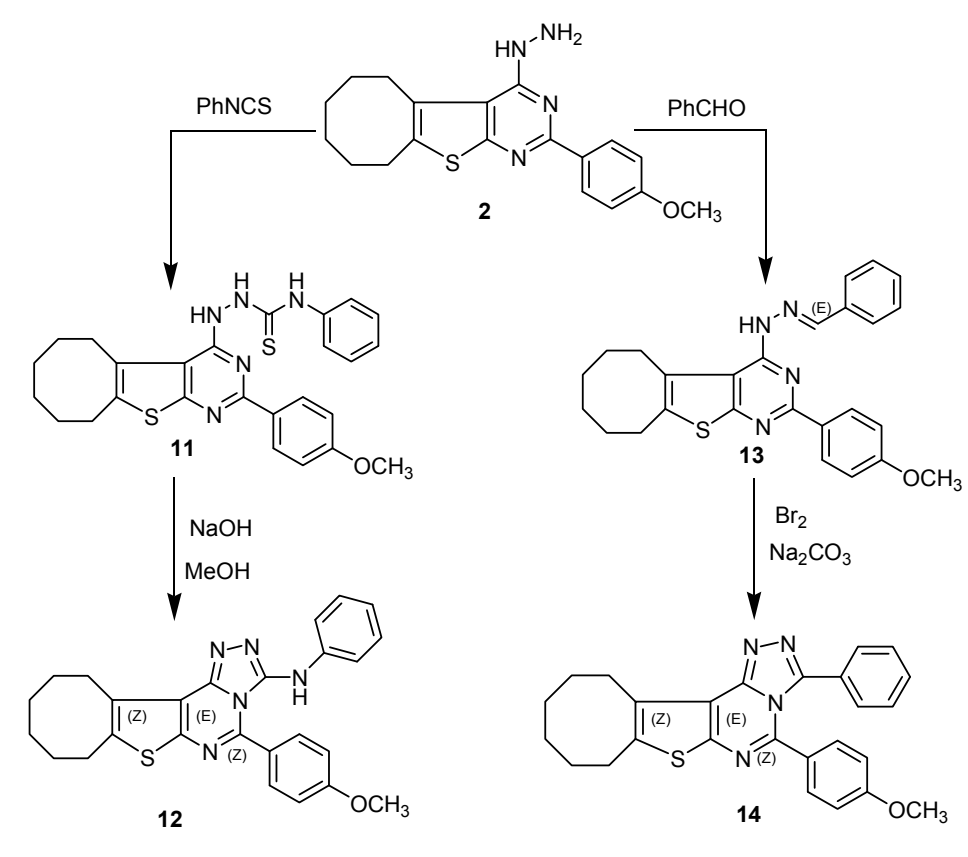

\subsection{Pharmacological Screening}

Two pharmacological activities namely; anti-inflammatory and ulcerogenic activities were tested despite their different biological receptors. Eleven representative compounds-2-10, 12 and 14-were evaluated as anti-inflammatory agents. Compounds $\mathbf{2}$ and $\mathbf{1 0}$ were also evaluated for ulcerogenic activity. The activities of these compounds are different according to the structure and function groups (Tables 1 and 2).

\subsubsection{Anti-inflammatory Activity}

The anti-inflammatory results after $1 \mathrm{~h}$ (percent inhibition of edema obtained by the reference drug and tested compounds, respectively) show that compounds 2, 3, 6, 9 and $\mathbf{1 0}$ possess strong anti-inflammatory activity $(42.3 \pm 1.1,37.2 \pm 1.3,34.5 \pm 1.2,31.2 \pm 1.2$ and $39.1 \pm 1.5 \%$ of inhibition, respectively), in comparison to that of indomethacin (44.7 $\pm 1.2 \%)$. Compounds $7, \mathbf{8}$ and 12 possess moderate anti-inflammatory activity $(20.8 \pm 1.4,21.3 \pm 1.2$ and $19.4 \pm 1.6 \%$ of inhibition, respectively), in comparison to that of indomethacin ( $44.7 \pm 1.2 \%)$. Compounds $\mathbf{4}, \mathbf{5}$ and $\mathbf{1 4}$ possess weak anti-inflammatory activity from $(16.5 \pm 1.3,11.7 \pm 1.1$ and $7.3 \pm 1.1 \%$ of inhibition, respectively), in comparison to that of indomethacin $(44.7 \pm 1.2 \%)$. The anti-inflammatory assay results (percent inhibition of edema obtained by the reference drug and tested compounds) after $2 \mathrm{~h}$ show that compounds $2, \mathbf{3}, \mathbf{6}, \mathbf{9}$ and $\mathbf{1 0}$ possess strong anti-inflammatory activity $(49.3 \pm 1.1,46.3 \pm$ $1.5,42.1 \pm 1.3,35.1 \pm 1.5$ and $48.4 \pm 1.2 \%$ of inhibition, respectively), in comparison to that of 
indomethacin $(52.4 \pm 1.2 \%)$. Compounds 7,8 and $\mathbf{1 2}$ possess moderate anti-inflammatory activity $(22.3 \pm 1.3,24.6 \pm 1.1$ and $21.3 \pm 1.3 \%$ of inhibition, respectively) in comparison to that of indomethacin $(52.4 \pm 1.2 \%)$. Compounds $\mathbf{4}, \mathbf{5}$ and $\mathbf{1 4}$ possess weak anti-inflammatory activity from $(17.2 \pm 1.1,13.2 \pm 1.2$ and $4.8 \pm 1.2 \%$ of inhibition, respectively) in comparison to that of indomethacin $(52.4 \pm 1.2 \%)$. The results of anti-inflammatory after $3 \mathrm{~h}$ show that compounds $2,3, \mathbf{6 ,} 9$ and 10 possess strong anti-inflammatory activity $(57.1 \pm 1.4,54.4 \pm 1.1,48.2 \pm 1.2,40.2 \pm 1.6$ and $55.6 \pm 1.1 \%$ of inhibition, respectively) in comparison to that of indomethacin $(61.2 \pm 1.3 \%)$. Compounds 7, 8 and 12 possess moderate anti-inflammatory activity $(25.1 \pm 1.4,26.5 \pm 1.2$ and $23.3 \pm$ $1.2 \%$ of inhibition, respectively) in comparison to that of indomethacin (61.2 $\pm 1.3 \%)$. Compounds $\mathbf{4 , 5}$ and 14 possess weak anti-inflammatory activity (from $19.3 \pm 1.2,15.2 \pm 1.5$ and $11.2 \pm 1.5 \%$ of inhibition, respectively) in comparison to that of indomethacin (61.2 $\pm 1.3 \%)$ (Table 1$)$.

Table 1. Anti-inflammatory activity of the synthesized compounds.

\begin{tabular}{|c|c|c|c|}
\hline \multirow{2}{*}{ Compound No. } & \multicolumn{3}{|c|}{ Edema inhibition (Means \pm E.M) ${ }^{\text {a,b }}(\%)$} \\
\hline & $1 \mathrm{~h}$ & $2 \mathrm{~h}$ & $3 \mathbf{h}$ \\
\hline 2 & $42.3 \pm 1.1$ & $49.2 \pm 1.2$ & $57.1 \pm 1.4$ \\
\hline 3 & $37.2 \pm 1.3$ & $46.3 \pm 1.5$ & $54.4 \pm 1.1$ \\
\hline 4 & $16.5 \pm 1.3$ & $17.2 \pm 1.1$ & $19.3 \pm 1.2$ \\
\hline 5 & $11.7 \pm 1.1$ & $13.2 \pm 1.2$ & $15.2 \pm 1.5$ \\
\hline 6 & $34.5 \pm 1.2$ & $42.1 \pm 1.3$ & $48.2 \pm 1.2$ \\
\hline 7 & $20.8 \pm 1.4$ & $22.3 \pm 1.3$ & $25.1 \pm 1.4$ \\
\hline 8 & $21.3 \pm 1.2$ & $24.6 \pm 1.1$ & $26.5 \pm 1.2$ \\
\hline 9 & $31.2 \pm 1.2$ & $35.1 \pm 1.5$ & $40.2 \pm 1.6$ \\
\hline 10 & $39.1 \pm 1.5$ & $48.4 \pm 1.2$ & $55.6 \pm 1.1$ \\
\hline 12 & $19.4 \pm 1.6$ & $21.3 \pm 1.3$ & $23.3 \pm 1.2$ \\
\hline 14 & $7.3 \pm 1.1$ & $9.8 \pm 1.2$ & $11.2 \pm 1.5$ \\
\hline Indomethacin & $44.7 \pm 1.2$ & $52.4 \pm 1.2$ & $61.2 \pm 1.3$ \\
\hline
\end{tabular}

${ }^{\mathrm{a}}$ Dose $5 \mathrm{mg} / \mathrm{kg}$ b.m(p.o.); ${ }^{\mathrm{b}} \mathrm{n}=6$.

\subsubsection{Ulcerogenicity}

Compounds $\mathbf{2}$ and $\mathbf{1 0}$ showed higher anti-inflammatory activities than the other compounds so they were also screened for their ulcerogenic activity at dose levels of 10, 50 and $100 \mathrm{mg} / \mathrm{kg}$ b.m. (Table 2). The tested compound $\mathbf{2}$ and $\mathbf{1 0}$ showed no ulcerogenic activity of 1.4 to $2.1 \mathrm{~mm}$.

Table 2. Ulcerogenic activities of compounds $\mathbf{2}$ and $\mathbf{1 0}$ (Gastric Ulceration in mice ${ }^{\mathrm{a}}$ ).

\begin{tabular}{cccc}
\hline \multirow{2}{*}{ Compd. No. } & \multicolumn{3}{c}{ Dose $(\mathbf{m g} / \mathbf{k g})^{\mathbf{a}}$} \\
\cline { 2 - 4 } & $\mathbf{1 0}$ & $\mathbf{5 0}$ & $\mathbf{1 0 0}$ \\
\hline Control & $0 / 6$ & $0 / 6$ & $0 / 6$ \\
2 & $0 / 6(0)$ & $0 / 6(0)$ & $0 / 6(0)$ \\
10 & $0 / 6(0)$ & $0 / 6(0)$ & $0 / 6(0)$ \\
Indomethacin & $3 / 6(1.4 \pm 0.2)^{\mathrm{b}, \mathrm{c}}$ & $5 / 6(1.9 \pm 0.2)^{\mathrm{b}, \mathrm{c}}$ & $6 / 6(2.1 \pm 0.2)^{\mathrm{b}, \mathrm{c}}$ \\
\hline
\end{tabular}

${ }^{a}$ number of mice lesions bigger than $0.5 \mathrm{~mm}$ in length per total no. of mice. ${ }^{b}$ mean ulcer lesions $\pm \mathrm{SEM}(\mathrm{mm})$ $(n=6)$ in parentheses. ${ }^{c}$ significant difference at $p \leq 0.05$ compared to the control. 


\subsubsection{Acute Toxicity}

To determine the acute toxicity of the compounds, the $\mathrm{LD}_{50}$ values for compounds $\mathbf{2}, \mathbf{3}, \mathbf{6}, \mathbf{9}$ and 10 were determined by injecting different gradual increased doses of the tested compounds into male adult mice, then calculating the dose corresponding to 50\% animal death (Table 3).

Table 3. Acute toxicity $\left(\mathrm{LD}_{50}\right)$ of some of synthesized compounds.

\begin{tabular}{cc}
\hline Compound No. & LD $_{\mathbf{5 0}}[\mathbf{m g} / \mathbf{k g}]$ * \\
\hline 2 & $2.514 \pm 0.012$ \\
3 & $2.131 \pm 0.013$ \\
6 & $2.047 \pm 0.012$ \\
9 & $1.832 \pm 0.012$ \\
10 & $2.341 \pm 0.011$ \\
Indomethacin & $1.817 \pm 0.015$ \\
\hline
\end{tabular}

* Significant difference at $p \leq 0.05$ compared to the control.

\subsection{Structure Activity Relationships}

Structure activity relationships based on the obtained results indicated that substitution of pyrimidine derivatives modulated the anti-inflammatory activity. Compounds 2, 3, 6, 9 and 10 have high anti-inflammatory activity due to the presence of electron-donating moieties which increase the pharmacological activity. The sequence of anti-inflammatory properties regarding substitution of electron-donating group in pyrimidine derivatives is as follows: hydrazine $>$ methyl $>$ cyanomethyl $>$ tetrazine $>$ amide as exhibited in compounds $\mathbf{2}>\mathbf{1 0}>\mathbf{3}>\mathbf{6}>\mathbf{9}$, respectively. Compounds 7, 8 and 12 have moderate anti-inflammatory activity due to the presence of electron-withdrawing moieties which decrease the pharmacological activity. The sequence of anti-inflammatory properties regarding substitution of electron-withdrawing groups in pyrimidine derivatives is: amide $>$ carbonyl adjacent to amide $>$ phenyl amine as exhibited in compounds $8>7>\mathbf{1 2}$, respectively. Also, compounds 4, 5 and 14 have weak anti-inflammatory activity due to the presence of electron-withdrawing moieties which strongly decrease the pharmacological activity. The sequence of anti-inflammatory properties regarding electron-withdrawing group substitution in pyrimidine derivatives is in this case: carbonyl of imidazole $>$ carbonyl of pyrimidine $>$ phenyl as exhibited in compounds $4>5>14$, respectively.

\section{Experimental}

\subsection{General}

Melting points were determined on open glass capillaries using an Electrothermal IA 9000 SERIES digital melting point apparatus (Electrothermal, Essex, UK) and are uncorrected. Elemental analyses were performed with all final compounds on an Elementar Vario EL, located at the Microanalytical Unit, National Research Centre, Cairo, Egypt and were found within $\pm 0.4 \%$ of the theoretical values. The IR spectra (KBr) were recorded on a FT IR-8201 PC spectrophotometer. The ${ }^{1} \mathrm{H}-\mathrm{NMR}$ spectra were measured with a Jeol FTGNM-EX 270, $270 \mathrm{MHz}$ instrument in DMSO- $d_{6}$ and the chemical shifts were recorded in $(\delta, \mathrm{ppm})$ relative to TMS. The mass spectra were run at $70 \mathrm{eV}$ with a Finnigan 
SSQ 7000 spectrometer using EI and the $m / z$ values are indicated in Dalton. TLC (silica gel, aluminum sheets $60 \mathrm{~F}_{254}$, Merck, Darmstadt, Germany) was used to follow the reactions.

\subsection{Chemical Synthesis}

2-(4-Methoxyphenyl)-4-hydrazinocycloocteno[4.5]thieno[2.3-d]pyrimidine (2). A mixture of 1 (3.5 g, $0.01 \mathrm{~mol})$ and hydrazine hydrate $(0.68 \mathrm{~g}, 0.01 \mathrm{~mol})$ in $n$-butanol $(20 \mathrm{~mL})$ was heated under reflux for $6 \mathrm{~h}$, the formed solid was filtered off and crystallized from ethanol to give $\mathbf{2}$ as a green powder (76\%); mp 235-237 ${ }^{\circ} \mathrm{C}$; IR (KBr, $\left.v, \mathrm{~cm}^{-1}\right): 3336-3420\left(\mathrm{NH}, \mathrm{NH}_{2}\right) ;{ }^{1} \mathrm{H}-\mathrm{NMR}$ (DMSO- $\left.d_{6}, \delta, \mathrm{ppm}\right): 1.28-1.93$ (m, 8H, $\left.4 \mathrm{CH}_{2}\right), 2.74-3.12\left(\mathrm{~m}, 4 \mathrm{H}, 2 \mathrm{CH}_{2}\right), 3.52\left(\mathrm{~s}, 3 \mathrm{H}, \mathrm{OCH}_{3}\right), 6.45\left(\mathrm{~s}, 2 \mathrm{H}, \mathrm{NH}_{2}, \mathrm{D}_{2} \mathrm{O}\right.$ exchangeable), 7.14-7.56 (m, 4H, Ar-H), $9.12\left(\mathrm{~s}, 1 \mathrm{H}, \mathrm{NH}, \mathrm{D}_{2} \mathrm{O}\right.$ exchangeable); MS: $m / z(\%): 354\left(\mathrm{M}^{+}, 31\right)$ and 216 (100, $\mathrm{M}^{+}-\mathrm{C}_{7} \mathrm{H}_{10} \mathrm{~N}_{2} \mathrm{O}$ ). Anal. calcd for $\mathrm{C}_{19} \mathrm{H}_{22} \mathrm{~N}_{4} \mathrm{OS}$ (354.43): C, 64.40; H, 6.21; N, 15.81; Found: C, 64.13; H, 6.02; N, 15.59.

5-(4-Methoxyphenyl)-3-cyanomethylcycloocteno[4.5]thieno[2.3-d]triazolo[1,2,4]-[4,5-a]pyrimidine (3). A mixture of 1 ( $3.5 \mathrm{~g}, 0.01 \mathrm{~mol})$ and cyanoacetyl hydrazine $(0.99 \mathrm{~g}, 0.01 \mathrm{~mol})$ in $n$-butanol $(30 \mathrm{~mL})$ was refluxed for $8 \mathrm{~h}$, the product obtained after cooling was crystallized from pet. ether (b.p. $40-60{ }^{\circ} \mathrm{C}$ ) to give 3 as a reddish-brown powder $(72 \%)$; mp $289-291^{\circ} \mathrm{C}$; IR $\left(\mathrm{KBr}, v, \mathrm{~cm}^{-1}\right): 2219(\mathrm{CN}) ;{ }^{1} \mathrm{H}-\mathrm{NMR}$ (DMSO- $\left.d_{6}, \delta, \mathrm{ppm}\right): 1.25-1.84\left(\mathrm{~m}, 8 \mathrm{H}, 4 \mathrm{CH}_{2}\right), 2.36-2.87\left(\mathrm{~m}, 4 \mathrm{H}, 2 \mathrm{CH}_{2}\right), 3.18\left(\mathrm{~s}, 3 \mathrm{H}, \mathrm{OCH}_{3}\right), 3.46(\mathrm{~s}$, $\left.2 \mathrm{H}, \mathrm{CH}_{2}\right), 7.21-7.63(\mathrm{~m}, 4 \mathrm{H}, \mathrm{Ar}-\mathrm{H})$; MS: $m / z(\%): 403\left(\mathrm{M}^{+}, 19\right)$ and $256\left(100, \mathrm{M}^{+}-\mathrm{C}_{9} \mathrm{H}_{9} \mathrm{NO}\right)$. Anal. calcd. for $\mathrm{C}_{22} \mathrm{H}_{21} \mathrm{~N}_{5} \mathrm{OS}$ (403.45): C, 65.50; H, 5.21; N, 17.36; Found: C, 65.24; H, 5.01; N, 17.12 .

Synthesis of Compounds $\mathbf{4}$ and $\mathbf{5}$

Amino acids, namely glycine and anthranilic acid $(0.01 \mathrm{~mol})$, respectively, were poured into a solution of 1 (3.5 g, $0.01 \mathrm{~mol})$ in ethanol $(20 \mathrm{~mL})$. The reaction mixture was refluxed for $5 \mathrm{~h}$ then, the solid separated was refluxed with acetic anhydride $(15 \mathrm{~mL})$ for $3 \mathrm{~h}$ and the product obtained after cooling was crystallized from a proper solvent to give $\mathbf{4}$ and $\mathbf{5}$, respectively.

5-(4-Methoxyphenyl)-3-oxo-cycloocteno[4.5]thino[2,3-d]-2H-pyrazolo[3,2-c]-pyrimidine (4). From ethanol, a brown powder (59\%); mp over $300{ }^{\circ} \mathrm{C}$; IR $\left(\mathrm{KBr}, v, \mathrm{~cm}^{-1}\right): 1730(\mathrm{C}=\mathrm{O}) ;{ }^{1} \mathrm{H}-\mathrm{NMR}\left(\mathrm{DMSO}-d_{6}, \delta, \mathrm{ppm}\right)$ : 1.36-1.78 (m, 8H, 4CH $), 2.23-2.75\left(\mathrm{~m}, 4 \mathrm{H}, 2 \mathrm{CH}_{2}\right), 2.91\left(\mathrm{~s}, 2 \mathrm{H}, \mathrm{CH}_{2}\right.$, imidazole), $3.18\left(\mathrm{~s}, 3 \mathrm{H}, \mathrm{OCH}_{3}\right)$ and 7.12-7.58 (m, 4H, Ar-H); MS: $m / z(\%): 379\left(\mathrm{M}^{+}, 24\right)$ and $216\left(100, \mathrm{M}^{+}-\mathrm{C}_{9} \mathrm{H}_{11} \mathrm{NO}_{2}\right)$. Anal. calcd. for $\mathrm{C}_{21} \mathrm{H}_{21} \mathrm{~N}_{3} \mathrm{O}_{2} \mathrm{~S}$ (379.43): C, 66.49; H, 5.54; N, 11.08; Found: C, 66.21; H, 5.19; N, 10.82 .

8-(4-Methoxyphenyl)-6-oxo-cycloocteno[4.5]thino[2,3-d]pyrimidino[4.3-b]quinazoline (5). From methanol, a yellow powder $(71 \%)$; mp over $250{ }^{\circ} \mathrm{C}$; IR $\left(\mathrm{KBr}, v, \mathrm{~cm}^{-1}\right): 1732(\mathrm{C}=\mathrm{O}) ;{ }^{1} \mathrm{H}-\mathrm{NMR}$ (DMSO- $d_{6}, \delta$, ppm): $1.31-1.54\left(\mathrm{~m}, 8 \mathrm{H}, 4 \mathrm{CH}_{2}\right), 1.91-2.14\left(\mathrm{~m}, 4 \mathrm{H}, 2 \mathrm{CH}_{2}\right), 2.91\left(\mathrm{~s}, 3 \mathrm{H}, \mathrm{OCH}_{3}\right)$ and 7.16-7.64 (m, 8H, Ar-H); MS: $m / z(\%)$ : $455\left(\mathrm{M}^{+}, 25\right) ; 264\left(100, \mathrm{M}^{+}-\mathrm{C}_{13} \mathrm{H}_{19} \mathrm{O}\right)$. Anal. calcd. for $\mathrm{C}_{26} \mathrm{H}_{23} \mathrm{~N}_{4} \mathrm{O}_{2} \mathrm{~S}(455.51)$ : C, 70.74; H, 5.21; N, 9.52; Found: C, 70.51; H, 5.01; N, 9.26.

5-(4-Methoxyphenyl)-cycloocteno[4.5]thieno[2.3-d]tetrazolo[4.5-e]pyrimidine (6). A solution of $\mathbf{1}$ $(3.5 \mathrm{~g}, 0.01 \mathrm{~mol})$ and sodium azide $(0.65 \mathrm{~g}, 0.01 \mathrm{~mol})$ in glacial acetic acid $(30 \mathrm{~mL})$ was refluxed for $3 \mathrm{~h}$. The product obtained after cooling was crystallized from benzene to give 6 as a green powder (59\%); 
mp 254-256 ${ }^{\circ} \mathrm{C}$; IR $\left(\mathrm{KBr}, v, \mathrm{~cm}^{-1}\right)$ : 1621-1435 $(\mathrm{C}=\mathrm{N}, \mathrm{C}=\mathrm{C}), 1281(\mathrm{~N}-\mathrm{N}=\mathrm{N}), 1136$ (tetrazole ring); ${ }^{1} \mathrm{H}-\mathrm{NMR}$ (DMSO-d $\left.6, \delta, \mathrm{ppm}\right): 1.43-1.85\left(\mathrm{~m}, 8 \mathrm{H}, 4 \mathrm{CH}_{2}\right), 2.87-2.92\left(\mathrm{~m}, 4 \mathrm{H}, 2 \mathrm{CH}_{2}\right), 3.15(\mathrm{~s}, 3 \mathrm{H}$, $\left.\mathrm{OCH}_{3}\right)$ and 7.26-7.38 (m, 4H, Ar-H); MS: m/z (\%); $365\left(\mathrm{M}^{+}, 42\right)$ and $216\left(100, \mathrm{M}^{+}-\mathrm{C}_{7} \mathrm{H}_{7} \mathrm{~N}_{3} \mathrm{O}\right)$. Anal. calcd. for $\mathrm{C}_{19} \mathrm{H}_{19} \mathrm{~N}_{5} \mathrm{OS}(365.41)$ : C, 62.46; H, 5.20; N, 19.17; Found: C, 62.14; H, 5.04; N, 19.03.

6-(4-Methoxyphenyl)-3,4-dioxo-2H-cycloocteno[4.5]thieno[2,3-d]triazino[1,2.4]-[3,4-a]pyrimidine (7). A solution of $2(3.50 \mathrm{~g}, 0.01 \mathrm{~mol})$ and diethyl oxalate $(1.46 \mathrm{~g}, 0.01 \mathrm{~mol})$ in ethanol $(30 \mathrm{~mL})$ was refluxed for $8 \mathrm{~h}$.; The solid obtained after cooling was filtered and crystallized from ethanol to give 7 as a reddish brown solid (64\%); mp over $300{ }^{\circ} \mathrm{C}$; IR $\left(\mathrm{KBr}, v, \mathrm{~cm}^{-1}\right): 3315(\mathrm{NH}), 1730(\mathrm{C}=\mathrm{O}$ amide, $\mathrm{C}-3$ of triazine), 1728 ( $\mathrm{C}=\mathrm{O}$ amide of $\mathrm{C}-4$ triazine); ${ }^{1} \mathrm{H}-\mathrm{NMR}$ spectrum (DMSO- $d_{6}, \delta$, ppm): $1.32-1.91$ $\left(\mathrm{m}, 8 \mathrm{H}, 4 \mathrm{CH}_{2}\right), 2.67-2.82\left(\mathrm{~m}, 4 \mathrm{H}, 2 \mathrm{CH}_{2}\right), 3.25\left(\mathrm{~s}, 3 \mathrm{H}, \mathrm{OCH}_{3}\right), 7.24-7.38(\mathrm{~m}, 4 \mathrm{H}, \mathrm{Ar}-\mathrm{H})$ and $10.17(\mathrm{~s}$, $1 \mathrm{H}, \mathrm{NH} ; \mathrm{D}_{2} \mathrm{O}$ exchangeable); MS: $m / z(\%) ; 408\left(\mathrm{M}^{+}, 23\right)$ and $337\left(100, \mathrm{M}^{+}-\mathrm{C}_{2} \mathrm{HNO}_{2}\right)$. Anal. calcd. for $\mathrm{C}_{21} \mathrm{H}_{20} \mathrm{~N}_{4} \mathrm{O}_{3} \mathrm{~S}$ (408.43): C, 61.76; H, 4.90; N, 13.72; Found: C, 61.32; H, 4.68; N, 13.43.

6-(4-Methoxyphenyl)-3-oxo-2,4-dihydrocycloocteno[4,5] thieno[2,3-d]triazino-[1,2,4][3,4-a]pyrimidine (8). A mixture of $2(3.50 \mathrm{~g}, 0.01 \mathrm{~mol})$ and chloroacetyl chloride $(1.13 \mathrm{~g}, 0.01 \mathrm{~mol})$ in pyridine $(20 \mathrm{~mL})$ was refluxed for $10 \mathrm{~h}$, then, poured on dil. $\mathrm{HCl}$. The solid product formed was crystallized from ethanol to give 8 as a brown powder (73\%); mp 284-286 ${ }^{\circ} \mathrm{C}$; IR $\left(\mathrm{KBr}, \mathrm{v}, \mathrm{cm}^{-1}\right)$ : $3315(\mathrm{NH}), 1725(\mathrm{C}=\mathrm{O})$; ${ }^{1} \mathrm{H}-\mathrm{NMR}$ (DMSO-d $\left.d_{6}, \delta, \mathrm{ppm}\right): 1.15-1.82\left(\mathrm{~m}, 8 \mathrm{H}, 4 \mathrm{CH}_{2}\right), 1.98-2.65\left(\mathrm{~m}, 4 \mathrm{H}, 2 \mathrm{CH}_{2}\right), 3.52(\mathrm{~s}, 3 \mathrm{H}$, $\left.\mathrm{OCH}_{3}\right), 3.81\left(\mathrm{~s}, 2 \mathrm{H}, \mathrm{CH}_{2}\right), 7.21-7.59(\mathrm{~m}, 4 \mathrm{H}, \mathrm{Ar}-\mathrm{H})$ and 9.01 (bs, 1H, NH; $\mathrm{D}_{2} \mathrm{O}$ exchangeable); MS: $m / z(\%): 394\left(\mathrm{M}^{+}, 31\right)$ and $230\left(100, \mathrm{M}^{+}-\mathrm{C}_{9} \mathrm{H}_{10} \mathrm{NO}_{2}\right)$. Anal. calcd. for $\mathrm{C}_{21} \mathrm{H}_{22} \mathrm{~N}_{4} \mathrm{O}_{2} \mathrm{~S}$ (394.45): C, 63.95; H, 5.58; N, 14.21; Found: C, 63.63; H, 5.32; N, 14.03.

5-(4-Methoxy)phenyl-3-oxo-2H-cycloocteno[4,5] thieno[2,3-d] [1,2,4]triazolo[4,5-a]pyrimidine (9). A mixture of $2(3.50 \mathrm{~g}, 0.01 \mathrm{~mol})$ and ethyl chloroformate $(1.08 \mathrm{gm}, 0.01 \mathrm{~mol})$ in pyridine $(20 \mathrm{~mL})$ was refluxed for $12 \mathrm{~h}$ then, poured on dil. $\mathrm{HCl}$. The solid obtained was crystallized from methanol to give 9 as a green powder $(54 \%) ; \mathrm{mp}>300{ }^{\circ} \mathrm{C}$; IR $\left(\mathrm{KBr}, v, \mathrm{~cm}^{-1}\right): 3320(\mathrm{NH}), 1731(\mathrm{C}=\mathrm{O}) ;{ }^{1} \mathrm{H}-\mathrm{NMR}$ $\left(\mathrm{DMSO}-d_{6}, \delta, \mathrm{ppm}\right): 1.32-1.72\left(\mathrm{~m}, 8 \mathrm{H}, 4 \mathrm{CH}_{2}\right), 2.19-2.41\left(\mathrm{~m}, 4 \mathrm{H}, 2 \mathrm{CH}_{2}\right), 2.84\left(\mathrm{~s}, 3 \mathrm{H}, \mathrm{OCH}_{3}\right), 6.24$ (bs, 1H, NH; $\mathrm{D}_{2} \mathrm{O}$ exchangeable), 7.08-7.31 (m, 4H, Ar-H),; MS : $m / z(\%) ; 380\left(\mathrm{M}^{+}, 19\right)$ and 216 (100, $\mathrm{M}^{+}-\mathrm{C}_{8} \mathrm{H}_{8} \mathrm{~N}_{2} \mathrm{O}_{2}$ ). Anal. calcd. for $\mathrm{C}_{20} \mathrm{H}_{20} \mathrm{~N}_{4} \mathrm{O}_{2} \mathrm{~S}$ (380.42): C, 63.15; H, 5.26; N, 14.73; Found: C, $62.89 ; \mathrm{H}, 5.04 ; \mathrm{N}, 14.49$.

5-(4-Methoxyphenyl)-3-methyl-2H-cycloocteno[4,5] thieno[2,3-d][1,2,4]triazolo-[4,5-a] pyrimidine (10) A solution of $2(3.50 \mathrm{~g}, 0.01 \mathrm{~mol})$ and acetic anhydride $(20 \mathrm{~mL})$ was heated under reflux for $2 \mathrm{~h}$. The solid obtained after cooling was crystallized from pet. ether to give $\mathbf{1 0}$ as a yellow powder (54\%); mp 272-274 ${ }^{\circ} \mathrm{C}$; IR $\left(\mathrm{KBr}, v, \mathrm{~cm}^{-1}\right): 1418-1432\left(\mathrm{C}=\mathrm{N}, \mathrm{C}=\mathrm{C}_{\mathrm{Ar}}\right) ;{ }^{1} \mathrm{H}-\mathrm{NMR}$ (DMSO- $\left.d_{6}, \delta, \mathrm{ppm}\right): 1.17-1.68(\mathrm{~m}$, $\left.8 \mathrm{H}, 4 \mathrm{CH}_{2}\right), 1.93-2.81\left(\mathrm{~m}, 4 \mathrm{H}, 2 \mathrm{CH}_{2}\right), 2.16\left(\mathrm{~s}, 3 \mathrm{H}, \mathrm{CH}_{3}\right), 2.21\left(\mathrm{~s}, 3 \mathrm{H}, \mathrm{OCH}_{3}\right)$ and $7.22-7.38(\mathrm{~m}, 4 \mathrm{H}, \mathrm{Ar}-\mathrm{H})$; MS: $m / z(\%) ; 379\left(\mathrm{M}^{+}, 56\right)$ and $231\left(100, \mathrm{M}^{+}-\mathrm{C}_{9} \mathrm{H}_{10} \mathrm{NO}\right)$. Anal. calcd. for $\mathrm{C}_{21} \mathrm{H}_{22} \mathrm{~N}_{4} \mathrm{OS}$ (379.49): C, 66.67; H, 5.82; N, 14.81; Found: C, 66.39; H, 5.57; N, 14.53.

$\mathrm{N}$-[2-(4-Methoxyphenyl)cycloocteno[4,5]thieno[2,3-d]pyrimidino-4-yl]-N-phenylthio-semicarbazide (11). A mixture of $2(3.50 \mathrm{~g}, 0.01 \mathrm{~mol})$ and phenyl isothiocyanate $(2.50 \mathrm{~mL})$ in ethanol $(30 \mathrm{~mL})$ was refluxed for $5 \mathrm{~h}$, the product formed after cooling was collected and crystallized from methanol to give 
11 as a reddish-brown solid (68\%); mp $189-191{ }^{\circ} \mathrm{C}$; IR (KBr, v, $\left.\mathrm{cm}^{-1}\right)$ : 3,342-3,415 (3 NH), 1,310 $(\mathrm{C}=\mathrm{S}) ;{ }^{1} \mathrm{H}-\mathrm{NMR}$ (DMSO- $\left.d_{6}, \delta, \mathrm{ppm}\right): 1.26-1.91\left(\mathrm{~m}, 8 \mathrm{H}, 4 \mathrm{CH}_{2}\right), 2.05-3.18\left(\mathrm{~m}, 4 \mathrm{H}, 2 \mathrm{CH}_{2}\right), 3.42$ (s, $\left.3 \mathrm{H}, \mathrm{OCH}_{3}\right), 7.21-7.46(\mathrm{~m}, 9 \mathrm{H}, \mathrm{Ar}-\mathrm{H}), 10.22\left(\mathrm{~s}, 1 \mathrm{H}, \mathrm{NH}\right.$ exchangeable with $\left.\mathrm{D}_{2} \mathrm{O}\right), 10.54(\mathrm{~s}, 1 \mathrm{H}, \mathrm{NH}$; $\mathrm{D}_{2} \mathrm{O}$ exchangeable) and $11.15\left(\mathrm{~s}, 1 \mathrm{H}, \mathrm{NH}, \mathrm{D}_{2} \mathrm{O}\right.$ exchangeable); MS: $m / z(\%) ; 489\left(\mathrm{M}^{+}, 31\right)$ and 216 (100, $\mathrm{M}^{+}-\mathrm{C}_{14} \mathrm{H}_{15} \mathrm{~N}_{3} \mathrm{OS}$ ). Anal. calcd. for $\mathrm{C}_{26} \mathrm{H}_{27} \mathrm{~N}_{5} \mathrm{OS}_{2}$ (489.59); C, 63.80; H, 5.52; N, 14.31; Found: C, 63.61; H, 5.25; N, 14.19.

5-(4-Methoxyphenyl)-3-anilinocycloocteno[4,5]thieno[2,3-d[1,2,4]triazolo[4,5-c]pyrimidine (12) A mixture of 11 (4.89 g, $0.01 \mathrm{~mol})$ and sod.hydroxide $(0.40 \mathrm{~g}, 0.01 \mathrm{~mol})$ in methanol $(20 \mathrm{~mL})$ was refluxed for $4 \mathrm{~h}$. The product formed after cooling was collected and crystallized from acetic acid to give $\mathbf{1 2}$ as white powder (69\%); mp 257-259 ${ }^{\circ} \mathrm{C}$; IR $\left(\mathrm{KBr}, v, \mathrm{~cm}^{-1}\right)$ : 3316-3350 $\left(\mathrm{NH} ; \mathrm{NH}_{2}\right) ;{ }^{1} \mathrm{H}-\mathrm{NMR}$ (DMSO- $d_{6}, \delta$, ppm): 1.32-1.74 (m, 8H, 4CH $), 1.89-2.61\left(\mathrm{~m}, 4 \mathrm{H}, 2 \mathrm{CH}_{2}\right), 2.95\left(\mathrm{~s}, 3 \mathrm{H}, \mathrm{OCH}_{3}\right), 7.31-7.75(\mathrm{~m}, 9 \mathrm{H}$, Ar-H) and 10.23 (bs, 1H, NH, $\mathrm{D}_{2} \mathrm{O}$ exchangeable); MS: $m / z(\%) ; 455\left(\mathrm{M}^{+}, 21\right)$ and $228(100$, $\mathrm{M}^{+}-\mathrm{C}_{13} \mathrm{H}_{13} \mathrm{~N}_{3} \mathrm{O}$ ). Anal. calcd for $\mathrm{C}_{26} \mathrm{H}_{25} \mathrm{~N}_{5} \mathrm{OS}$ (455.52): C, 68.57; H, 5.49; N, 15.38; Found: C, 68.21; $\mathrm{H}, 5.16 ; \mathrm{N}, 15.14$.

2-(4-Methoxyphenyl)-4-(N-benzylidenehydrazino)cycloocteno[4,5]thieno2,3-d]pyrimidine (13) A mixture of $2(3.50 \mathrm{~g}, 0.01 \mathrm{~mol})$ and benzaldehyde $(2.50 \mathrm{~mL})$ in ethanol $(20 \mathrm{~mL})$ was heated under reflux for $2 \mathrm{~h}$ then cooled, The separated solid was filtered, dried and crystallized from pet. ether (b.p. $40-60{ }^{\circ} \mathrm{C}$ ) to afford 13 as a green powder (48\%); mp $174-176{ }^{\circ} \mathrm{C}$; IR (KBr, $\left.v, \mathrm{~cm}^{-1}\right)$ : $3350(\mathrm{NH}) ;{ }^{1} \mathrm{H}-\mathrm{NMR}$ (DMSO- $d_{6}$, $\delta$, ppm): 1.25-1.82 (m, 8H, 4CH $), 3.06-3.14\left(\mathrm{~m}, 4 \mathrm{H}, 2 \mathrm{CH}_{2}\right), 3.32\left(\mathrm{~s}, 3 \mathrm{H}, \mathrm{OCH}_{3}\right), 3.65(\mathrm{~s}, 1 \mathrm{H}$, $\mathrm{CH}=\mathrm{N}), 7.21-7.78(\mathrm{~m}, 9 \mathrm{H}, \mathrm{Ar}-\mathrm{H})$ and $10.42\left(\mathrm{bs}, 1 \mathrm{H}, \mathrm{NH} ; \mathrm{D}_{2} \mathrm{O}\right.$ exchangeable); $\mathrm{MS}: \mathrm{m} / z(\%) ; 442\left(\mathrm{M}^{+}\right.$, 27) and $323\left(100, \mathrm{M}^{+}-\mathrm{C}_{7} \mathrm{H}_{7} \mathrm{~N}_{2}\right)$. Anal. calcd. for $\mathrm{C}_{26} \mathrm{H}_{26} \mathrm{~N}_{4} \mathrm{OS}$ (442.51): C, 70.58; H, 5.88; N, 12.67; Found: C, 70.31; H,5.64; N, 12.39 .

5-(4-Methoxyphenyl)-4-phenylcycloocteno[4,5]thieno[2,3-d][1,2,4]triazolo[4,5-c]pyrimidine (14) To a stirred mixture of 13 (4.42 g, $0.01 \mathrm{~mol})$ and anhydrous sodium bicarbonate (1.68 g, $0.02 \mathrm{~mol})$ in chloroform $(20 \mathrm{~mL})$ bromine $(1.50 \mathrm{~mL})$ was added. The mixture was stirred at room temperature for $5 \mathrm{~h}$, then left to stand overnight, and evaporated under vacuum. The residue was triturated with ice-cold water, and the product formed was filtered, washed with water, dried and crystallized from ethanol to give 14 as a reddish brown solid $(63 \%)$; mp over $300{ }^{\circ} \mathrm{C}$; IR $\left(\mathrm{KBr}, v, \mathrm{~cm}^{-1}\right): 1620-1435\left(\mathrm{C}=\mathrm{N}_{\mathrm{Ar}}\right.$, $\left.\mathrm{C}=\mathrm{C}_{\mathrm{Ar}}\right) ;{ }^{1} \mathrm{H}-\mathrm{NMR}\left(\mathrm{DMSO}-d_{6}, \delta, \mathrm{ppm}\right): 1.19-1.72\left(\mathrm{~m}, 8 \mathrm{H}, 4 \mathrm{CH}_{2}\right), 2.94-3.22\left(\mathrm{~m}, 4 \mathrm{H}, 2 \mathrm{CH}_{2}\right), 3.41$ (s, $\left.3 \mathrm{H}, \mathrm{OCH}_{3}\right)$ and 7.25-7.64 (m, 9H, Ar-H); MS: $m / z(\%)$; $440\left(\mathrm{M}^{+}, 42\right)$ and $256\left(100, \mathrm{M}^{+}-\mathrm{C}_{13} \mathrm{H}_{12} \mathrm{O}\right)$. Anal. calcd. for $\mathrm{C}_{26} \mathrm{H}_{24} \mathrm{~N}_{4} \mathrm{OS}(440.50)$; C, 70.91; H, 5.45; N, 12.72; Found: C, 70.65; H, 5.21; N, 12.42 .

\subsection{Pharmacological Screening}

\subsubsection{Animals}

Female albino mice (16-18 g) and Sprague Dawley mice (100 g) obtained from the Theodor Bilharz Research Institute (TBRI, Giza city, Egypt) were used. Approval of the institutional animal ethical committee for the animal studies was obtained from the Office of Environmental Health and 
Radiation Safety, ACUC Protocol 1096-5. The animals were maintained according to accepted standards of animal care.

\subsubsection{Anti-inflammatory Activity}

Newly synthesized thienopyrimidine derivatives were dissolved in $0.5 \%$ carboxymethyl cellulose (CMC) as a homogeneous solution and administered intraperitonneally (i.p.). One hundred and eight rats were divided into eighteen groups, each group consisting of six animals. Anti-inflammatory activity of the compounds was studied in mice using carrageenan induced edema. A suspension of the tested compound and the reference drug, indomethacin in aqueous solution was administered orally at a dose $5 \mathrm{mg} / \mathrm{kg}$. Control animals were treated with $0.5 \% \mathrm{CMC}$ only. After $30 \mathrm{~min}, 0.1 \mathrm{~mL}$ of freshly prepared $1.0 \%$ carrageenan solution (in formol saline) was injected into the sub-plantar region of the right hind paw according to Hernandez-Perez [35]. The right paw volume was measured using a digital plethysmometer (Model 7150, Ugo Basile, Varese, Italy), directly before and after 1, 2, 3 h, intervals after administration of the tested compounds.

\subsubsection{Ulcerogenic Activity}

Seventy-two mice were divided into twelve groups. Ulcerogenic activity was evaluated after oral administration of the tested compounds or indomethacin at doses of 10,50 , and $100 \mathrm{mg} / \mathrm{kg}$. Control mice received $0.5 \%$ CMC. Food but not water was removed $24 \mathrm{~h}$ before administration of the tested compounds. After $6 \mathrm{~h}$, the mice were sacrificed; the stomach was removed and opened along the greater curvature, washed with distilled water and cleaned gently by dipping in saline. The mucosa damage for each stomach was examined using a stereoscopic microscope and compared with the reference drug indomethacin according to the reported procedure [36].

\subsubsection{Acute Toxicity}

The median lethal doses $\left(\mathrm{LD}_{50}\right)$ of the most active compounds $\mathbf{2}, \mathbf{3}, \mathbf{6 , 9}$ and $\mathbf{1 0}$ were determined in mice [37]. Groups of male adult mice, each of six animals, were injected i.p. with graded doses of each of the test compounds. The percentage of mortality in each group of animals was determined $24 \mathrm{~h}$, after injection. Computation of $\mathrm{LD}_{50}$ was processed by a graphical method.

\subsubsection{Statistical Analysis}

Assay results are shown as mean \pm SE. Statistical analyses were carried out with Sigma Plot software (SPSS Inc., Chicago, IL, USA). One-way analysis of variance (ANOVA) followed by Tukey's post test was used to assess the presence of significant differences. Differences were considered statistically significant at $p \leq 0.05$.

\section{Conclusions}

The objective of the present study was to synthesize and investigate the anti-inflammatory activities of some new thienopyrimidine derivatives. The starting material $\mathbf{1}$ was used to synthesize the following compounds: hydrazine thinopyrimidine $\mathbf{2}$, triazolothinopyrimidine $\mathbf{3}$, benzopyrimidinothieno 
pyrimidine 5 and tetrazolothienopyrimidine 6. Compound $\mathbf{2}$ was used to synthesize the triazino- and triazolothinopyrimidine derivatives 7, 8,9 and 10, respectively. Also, compound 2 was carried out to synthesize of triazolothinopyrimidine derivatives 12 and $\mathbf{1 4}$. The newly synthesized compounds $\mathbf{2 - 1 0}$, 12 and 14 were screened for their anti-inflammatory activity compared to indomethacin which was used as reference drug. Compounds 3, 6, 9 and $\mathbf{1 0}$ possess strong anti-inflammatory activity, and compounds 8,7 and 12 possess moderate anti-inflammatory activity, while compounds 4,5 and 14 possess weak anti-inflammatory activity.

\section{Acknowledgements}

The Authors extend their appreciation to the Deanship of Scientific Research at King Saud University for funding the work through the research group project No. RGP-VPP-0172.

\section{Conflicts of Interest}

The authors declare no conflict of interest.

\section{References}

1. Al-Mohizea, A.M.; Al-Omar, M.A.; Abdalla, M.M.; Amr, A.E. 5 $\alpha$-Reductase inhibitors, antiviral and anti-tumor activities of some steroidal cyanopyridinone derivatives. Int. J. Biol. Macromol. 2012, 50, 171-179.

2. Abdalla, M.M.; Al-Omar, M.A.; Bhat, M.A.; Amr, A.E.; Al-Mohizeae, A.M. Steroidal pyrazolines evaluated as aromatase and quinone reductase-2 inhibitors for chemoprevention of cancer. Int. J. Biol. Macromol. 2012, 50, 1127-1132.

3. Bahashwan, S.A.; Al-Harbi, N.O.; Fayed, A.A.; Amr, A.E.; Shadid, K.A.; Alalawi, A.M.; Bassati, I.M.S. Synthesis and pharmacological evaluation of novel triazolo[4,3-b]pyrazolo[3,4c]pyridazine derivatives. Int. J. Biol. Macromol. 2012, 51, 7-17.

4. Al-Harbi, N.O.; Bahashwan, S.A.; Fayed, A.A.; Aboonq, M.S.; Amr, A.E. Anti-parkinsonism, hypoglycemic and anti-microbial activities of some new poly ring heterocyclic candidates. Int. J. Biol. Macromol. 2013, 57, 165-173.

5. Alanazi, A.M.; Al-Omar, M.A.; Abdulla, M.M.; Amr, A.E. Anti-arthritic and immune-suppressive activities of substituted triterpenoidal candidates. Int. J. Biol. Macromol. 2013, 58, 245-252.

6. Clark, J.; Shohhet, M.S.; Korakas, D.; Varvounis, G. Synthesis of thieno \{2,3-d]pyrimidines from 4,6-di chloropyrimidin-5-carbaldehydes. J. Heterocycl. Chem. 1993, 30, 1065-1072.

7. Kogowwra, I.Y.; Yimatsusita, N.N.; Pfkador, J.K. Synthesis of substituted 2,4-dioxo thieno pyrimidin-1-acetic acid and their evalution as aldose reductase inhibitors. Eur. J. Med. Chem. 1993, 28, 769-781.

8. Tozkoparan, B.; Ertan, M.; Kelicen, P.; Demirdamar, R. Synthesis and anti-inflammatory activities of some thiazolo[3,2-a] pyrimidine derivatives. II Farmaco. 1999, 54, 588-593.

9. Kurasawa, Y.; Mitomi, T.; Okamoto, Y.; Kim, H.S. A new tautomeric Zwitterion form of 2-acylamino-4H-1,3,4-oxadiazino[5,6-b]quinoxalines in solution. J. Heterocycl. Chem. 1998, 35, $1333-1338$. 
10. Nargund, L.V.G.; Badiger, V.V.; Yarnal, S.U. Synthesis and antibacterial activity of substituted 4-Aryloxypyrimido[5,4-c]cinnolines. Eur. J. Med. Chem. 1994, 29, 245-247.

11. Vanlaar, M.; Volerts, E.; Verbaten, M. Subchronic effect of the GABA-agonist lorazepan and the 5-HT2A/2C antagonist ritanserin on driving performance, Slow wave sleep and daytime sleepiness in healthy volunteers. Psychopharmacology 2001, 154, 189-197.

12. Danel, K.; Pedersen, E.B.; Nielsen, C. Synthesis and anti-HIV-1 activity of novel 2,3- dihydro7H-thiazolo[3,2-a]pyrimidin-7-ones. J. Med. Chem. 1998, 41, 191-198.

13. Vijesh, A.M.; Isloor, A.M.; Shetty, P.; Sundershan, S.; Fun, H.K. New pyrazole derivatives containing 1,2,4-triazoles and benzoxazoles as potent antimicrobial and analgesic agents. Eur. J. Med. Chem. 1962, 10, 410-415.

14. Amr, A.E.; Hegab, M.I.; Ibrahim, A.A.; Abdalah, M.M. Synthesis and reactions of some fused oxazinone, pyrimidinone, thiopyrimidnone and triazinone derivatives with thiophene ring as analgesic, Anticonvulsant and antiparkinsonian agents. Monatsh. Chem. 2003, 134, 1395-1409.

15. Abou-Ghalia, M.H.; Amr, A.E. Synthesis and investigation of a new cyclo-( $\mathrm{N}^{\alpha}$-dipicolinoyl)pentapeptide of a breast and CNS cytotoxic activity and an ionophoric specifity. Amino Acids 2004, 26, 283-289.

16. Hammam, A.G.; Fahmy, A.F.M.; Amr, A.E.; Mohamed, A.M. Synthesis of novel tricyclic heterocyclic compounds as potential anticancer agents using chromanone and thiochromanone as synthons. Ind. J. Chem. 2003, 42B, 1985-1993.

17. Amr, A.E.; Ali, K.A.; Abdalla, M.M. Cytotoxic, Antioxidant activities and structure activity relationship of some newly synthesized terpenoidal oxaliplatin analogs. Eur. J. Med. Chem. 2009, 44, 901-907.

18. Fayed, A.A.; Hosni, H.M.; Flefel, E.M.; Amr, A.E. Synthesis and pharmacological activity of some new thieno[2,3-d]pyrimidine and pyrimidopyrazolothieno pyrimidine derivatives. World $J$. Chem. 2009, 4, 58-65.

19. Hafez, H.N.; Abbas, H.A.; El-Gazzar, A.R. Synthesis and evaluation of analgesic, anti-inflammatory and ulcerogenic activities of some triazolo- and 2-pyrazolylpyrido[2,3-d]-pyrimidines. Acta Pharm. 2008, 58, 359-78.

20. Bahashwan, S.A. Pharmacological studies of some pyrimidino derivatives. Afr. J. Pharm. Pharmaco. 2011, 5, 527-531.

21. Singh, N.; Nargund, S.L.; Rashmi, P.; Nargund, L.V.G. Synthesis and antibacterial and anti-inflammatory activity of 4-substituted-thieno[2,3-d]pyrimidines. Chem. Sin. 2012, 3, 198-203.

22. Hassan, N.A.; Hegab, M.I.; Rashad, A.E.; Fahmy, A.A.; Abdel-Megeid, F.M. Synthesis and antimicrobial activity of some cyclic and acyclic nucleosides of thieno[2,3-d]pyrimidines. Nucleos. Nucleot. Nucleic Acids 2007, 26, 379-390.

23. Prachayasittikul, S.; Worachartcheewan, A.; Nantasenamat, C.; Chinworrungsee, M.; Sornsongkhram, N.; Ruchirawat, S.; Prachayasittikul, V. Synthesis and structure-activity relationship of 2-thiopyrimidine-4-one analogs as antimicrobial and anticancer agents. Eur. J. Med. Chem. 2011, 46, 738-742.

24. Mosharef, M.D.; Khandker, M.D.; Rahman, M.; Kamrul, H.M.D.; Hossain, M.I.; Abu Naser, M. Synthesis and antimicrobial evaluation of some new thienopyrimidine derivatives. Acta Pharm. 2006, 5, 441-450. 
25. Shetty, N.S.; Lamani, R.S.; Khazi, I.A.M. Synthesis and antimicrobial activity of some novel thienopyrimidines and triazolothienopyrimidines. J. Chem. Sci. 2009, 121, 301-307.

26. Flefel, E.M.; Abdel-Mageid, R.E.; Tantawy, W.A.; Ali, M.A.; Amr, A.E. Heterocyclic compounds based on 3-(4-bromophenyl) azo-5-phenyl-2(3H)-furanone: anti-avian influenza virus (H5N1) activity. Acta Pharm. 2012, 62, 593-606.

27. Ramakrishna, V.S.; Kambhampati, R.S.; Kothmirkar, P.; Arepalli, S.; Reddy, G.N. Convenient and efficient synthesis of some novel fused thieno pyrimidines using Gewald's Reaction. Synth. Commun. 2011, 41, 2835-2851.

28. Abdel-Hafez, N.A.; Mohamed, A.M.; Amr, A.E.; Abdalla, M.M. Antiarrhythmic activities of some newly synthesized tricyclic and tetracyclic thienopyridine derivatives. Sci. Pharm. 2009, 77, 539-553.

29. Mojahidi, S.; Rakib, E.; Sekkak, H.; Abouricha, S.; Benchat, N.; Mousse, H.A.; Zyad, A. Synthesis and in vitro cytotoxic evaluation of novel pyridazin-4-one derivatives. Arch. Pharm. Chem. Life Sci. 2010, 343, 310-313.

30. Butnariu, R.M.; Mangalagiu, I.I. New pyridazine derivatives: Synthesis, Chemistry and biological activity. Bioorg. Med. Chem. 2009, 17, 2823-2829.

31. Guan, L.; Sui, X.; Deng, X.Q.; Quan, Y.C.; Quan, Z.S. Synthesis and anticonvulsant activity of a new 6-alkoxy[1,2,4]triazolo[4,3-b]pyridazine. Eur. J. Med. Chem. 2010, 45, 1746-1752.

32. Nagawade, R.R.; Khanna, V.V.; Bhagwat, S.S.; Shinde, D.B. Synthesis of new series of 1-aryl1,4-dihydro-4-oxo-6-methylpyridazine-3-carboxylic acid as potential antibacterial agents. Eur. J. Med. Chem. 2005, 40, 1325-1330.

33. Rao, A.; Balzarini, J.; Cafbone, A.; Chimirri, A. 2-(2,6-Dihalophenyl)-3-(pyrimidin-2-yl)-1,3thia-zolidin-4-ones as non nucleoside HIV-1 reverse transcriptase. Antivir. Res. 2004, 63, 79-84.

34. Abdel-Fattah, B.; Kandeel, M.M.; Abdel-Hakeem M.; Fahmy, Z.M. Synthesis of certain fused thienopyrimidines of biological interest. J. Chin. Chem. Soc. 2006, 53, 403-412.

35. Hernadezperez, M.; Rabanal, R.M.; Delatorre, M.C.; Rodriguez, B. Analgesic, Anti-inflammatory, Antipyretic and haematological effects of aethiopinone, an o-naphthoquinone diterpenoid from Salvia aethiopis roots and two hemisynthetic derivatives. Planta Med. 1995, 61, 505-509.

36. Ikuta, H.; Shirota, H.; Kobayshi, S.; Yamagishi, Y.; Yamada, K.; Yamatsu I.; Katayama, K. Synthesis and antiinflammatory activities of 3-(3,5-ditertbutyl-4-hydroxybenzylidene)-pyrrolidin2-ones. J. Med. Chem. 1987, 30, 1995-1998.

37. Sztaricskai, F.; Takacs, E.I.; Pusztai, F.; Szabo, G.; Csipo, I. Antiulcer effect of 5-amino salicylic acid. Arch. Pharm. Med. Chem. 1999, 332, 321-326.

Sample Availability: Samples of the compounds are available from the authors.

(C) 2013 by the authors; licensee MDPI, Basel, Switzerland. This article is an open access article distributed under the terms and conditions of the Creative Commons Attribution license (http://creativecommons.org/licenses/by/3.0/). 